MASS RADIOGRAPHY: A NEW WEAPON AGAINST TUBERCULOSIS

\author{
By BRIAN C. THOMPSON, M.A., M.D. (Camb.) \\ (Tuberculosis Officer, Middlesex County Council, formerly Dorothy Temple Cross Research \\ Fellow in Tuberculosis)
}

Mass-radiography is approximately twenty years old. The earliest application of X-rays to the chests of apparently healthy persons in selected social groups, such as that of Chadwick in I924 was for research or epidemiological purposes rather than as a practical measure of casefinding. The method continued to be used by research organisations, especially in the U.S.A., and a mass of valuable epidemiological material rapidly accrued. It became clearly established that pulmonary tuberculosis can arise and spread without manifest symptoms, and that such disease may on occasion proceed to caseation, excavation, and subsequent healing, presenting at a later date gross multiple areas of fibrosis and calcification in an individual who has at no time been conscious of ill-health.

Such observations were of immense value in building up the sum of our present knowledge of the pathology of tuberculosis, but the scope of the method was, by its relatively large cost, always limited to researchers whose results were not necessarily judged by financial standards. Technical improvements in the fluoroscopic screen allowed Fellows (r934) to examine, with an accuracy comparable with that of X-ray photography, large numbers of employees of the Metropolitan Life Assurance Company, at no more cost than the original price of the machine, the current used in operating it, and his own salary. The fluoroscopic method remains, however, a heavy strain on the operator, and must be expected to miss a proportion of small soft lesions; neither does it provide a permanent record. In 1936 the Powers Rapid Paper method introduced a drastic cut both in cost of materials and in time consumed per examinee, while the full-sized image on paper was accurate enough to satisfy all but the most fastidious.

Parallel technical advances, however, had meanwhile brought us the fluoroscopic image photographed on cinematograph film. X-rays could be taken in this way as rapidly as by the Powers method and rather more cheaply, and when viewed by projection on to a screen were found to reach a remarkable degree of accuracy. This so-called "miniature" method is the most popular at present. There is some divergence of opinion as to the best size of film, but? $35 \mathrm{~mm}$. has become standard in this country. Films can be taken comfortably at the rate of 500 per day, and interpreted by a radiologist at the rate of 300-400 per hour; abnormal findings or technical errors are verified by re-takes on full-sized film, averaging about 5 per cent of total examinations. The cost of the whole procedure depends obviously on much more than the price of film consumed, which is very small. Capital charges on apparatus and current overheads, of which the salaries of the operating team are a large item, largely determine the cost of each individual examination. The more X-rays taken, the fewer the re-takes necessitated by bad technique, and the steadier the rate of employment of the unit-the more economical is the method. Working at a reasonable rate over a year, a fair figure for such examination has been computed at about a shilling. So the method, while much less costly than any hitherto devised, still demands an expenditure per capitum equivalent to a packet of twenty Woodbines, a comparison which perhaps enables the question of cost to be viewed in proper perspective.

\title{
Miniature Mass-radiography in England
}

The first apparatus to be released for civilian use in this country was put into service by the Medical Research Council in March 1943, in a series of surveys in which I myself took part. Up to the time of writing, the following persons have been examined : 4,720 employees of a modern factory, Io,903 Civil Servants, I,800 inmates of a mental hospital, and 6,000 employees of a Government Ordnance establishment. Results are so far available only from the first and second groups, each of which gave almost an equal yield of significant pulmonary tuberculosis, namely 0.4 per cent of lesions requiring treatment, and 0.9 per cent not definitely active, but requiring observation by serial X-ray films.

The $5^{6}$ persons found to have previously unsuspected tuberculosis were forthwith provided with the means to control and if possible cure their disease; their danger to the community was promptly neutralised to the best of our present therapeutic and hygienic capacity. Several 
have speedily reached the stage of quiescence and have returned to work. Those still unfit for work are eligible for financial help administered in a quite unobjectionable manner under the new Government Allowance Scheme.

It is difficult to deny that this was a valuable achievement on behalf both of the individual and of those who have been saved from infection by him. Against its high total cost in $£ \mathrm{~s}$. d. must be offset, not only the cost, amounting to anything between $£ I 50$ and $£ 2,000$, of sanatorium care required by the average phthisical life, but also a loss to the community of productive power possibly much higher in potential and a cumulative loss, on the same basis, for each secondary case occurring by the spread of infection.

Mass-radiography pays dividends. This was obviously recognised by the multitude of hardheaded American industrialists who, under no Government pressure, with little regard for public opinion, and no great need to appease their employees, and without overpowering interest in welfare, competed with each other for radiological units long before man-power became a war-time scarcity.

\section{Alternative Methods of Mass Examination}

If there were any cheaper way of doing the job, doubtless these same interests would have adopted it. Dr. Brailsford, who writes elsewhere in this issue, has advocated as an alternative method of detecting latent phthisis the mass examination of sputum specimens. If this suggestion is to be taken seriously-and, leaving aside its many technical and administration objections, it is difficult to perceive any advantage that it possesses over mass-radiography even in cheapness-it is clear that such a method would detect only a small proportion of persons with significant tuberculosis. Of the $5^{6}$ cases described above, only 13 had grossly positive sputum, I9 others had T.B. on laryngeal swab or gastric-lavage culture, and the remainder were bacteriologically negative. These last were, moreover, typical of the "early" or minimal disease which has so good a prognosis when treated promptly. It is no argument to say that some of them also have a good prognosis when untreated, or when undiscovered. We know that this is so, for mass-radiography detects healed tuberculosis in persons who have no history of illness. But we also know that a high proportion of these minimal lesions advance insidiously and produce symptoms only when they reach an advanced stage. If their prognosis is improved by early discovery-and all phthisiologists agree that the results of treatment depend, more than any other factor, on the quantitative degree of lung involvement-then it is at least highly probable that they are worth detecting.

As a further claim in favour of mass sputum examinations, Dr. Brailsford alleges that a significant proportion of persons produce T.B. in their sputum without X-ray evidence of disease. This traditional gibe of the old school of wooden stethoscopes comes strangely from a radiologist. It has never been substantiated in other than a handful of freak cases, and can usually be traced to poor X-ray technique or a clerical error in the bacteriological department. If Dr. Brailsford has had as many of these cases as he suggests (though he gives no figures) it is his duty to publish them in detail since his experience apparently differs from the rest of the civilised world.

The accuracy of the miniature X-ray apparatus cannot seriously be impugned. Trail (I943) claims that it can even detect lesions missed in full-sized films. Its ability to distinguish between active and healed tuberculosis is, however, no greater than that of pure radiology in general, and the final decision in any given case may. depend on comparison of serial films taken at 3- or 6-monthly intervals. This method of radiological diagnosis was employed in 0.9 per cent of cases with doubtful activity. The 0.4 per cent who had active disease requiring treatment were so diagnosed on radiological, bacteriological and clinical evidence, including estimation of the blood-sedimentation rate. The patient's account of his symptoms was often important. Twenty-nine of the 56 "treatment" cases confessed on questioning to symptoms of cough or lassitude or chest pain. This does not mean, however, that they had consulted, or intended to consult, their private doctor on this account, and indeed only 8 of the 29 had done so. To have a cough is one thing-which of us does not from time to time have a cough?-but to seek medical advice, so giving the opportunity for disease to be discovered, is quite another. This distinction is important, for it has been claimed (Toussaint, I943) that the bulk of the nation's consumptives can be detected by private doctors referring for.X-ray all patients with persistent respiratory symptoms. This is, of course, not mass-radiography, but merely a concentrated 
application of a diagnostic procedure long in existence and effectively practised in areas where the local Tuberculosis Dispensary has wisely changed its name to Chest Clinic (as in Middlesex), or has similarly avoided the average citizen's aversion to the word tuberculosis, and where a good radiological examination and opinion is promptly available.

It is a programme which must still miss a large number of people with advanced tuberculosis who do not take their symptoms to a doctor, not necessarily because they have no symptoms, but often because their symptoms seem to be trivial. No amount of proper education can teach a nation of coughers like the British that one cough is different from another, for at present this fine distinction is not clear even to their medical advisers.

Even if every cougher were X-rayed, the phthisical ones would be diagnosed relatively late, for it is abundantly clear that early phthisis is for practical purposes asymptomatic and can only be detected by routine examination. This was very clearly demonstrated long ago by the routine radiological examination of home-contacts, and all Tuberculosis Officers will testify to the success of this well-established procedure in picking up early cases with, generally speaking, a good prognosis. Since the majority of the population are not home contacts, a similar routine technique must be available for the early, symptomless case of phthisis among them, infected by contact with persons unknown. For this purpose our best instrument is mass-radiography, and for mass-radiography there is no substitute.

\section{Re-examination}

Mass-radiography by the minature method of the whole population of this country susceptible to phthisis is capable of being achieved within a few years from now. Re-examination of persons initially found negative is, however, quite another matter. Dr. Brailsford, in calculations apparently aimed at reducing mass-radiography to absurdity, insists that for it to be of any value the whole population would have to be X-rayed every three months, an obviously prodigious proposition. This he bases on a hypothesis that the incubation period of tuberculosis is between 4 and 6 weeks: here he either misunderstands or misrepresents the well-known ante-allergic period between infection by tubercle bacilli and the development of reaction to the tuberculin test, a phenomenon which bears no constant relationship to the pathogenesis of phthisis. The true incubation period of pulmonary tuberculosis is unknown. What is not unknown, however, is that re-examination of persons initially X-ray negative, from a selected group of those continuing in close contact with a sputum-positive associate, is so unprofitable that it can practically. be neglected (Seigal, I94I).

It is not necessary, then, to budget at the outset for more than a single examination of each individual. Children below the age of fifteen can probably also be excluded; significant phthisis is rare among them, and such tuberculous lesions as may occasionally be discovered are either benign or so rapidly fatal as to be not amenable to treatment.

\section{What to do with the cases discovered}

Mass-radiography thus becomes a practical proposition on the diagnostic side. Fears have been expressed that, so far from not detecting cases of tuberculosis, it may be lead to the detection of more cases than can be dealt with in existing conditions. Sanatorium facilities already overstrained by War-time stringency and an increase in cases diagnosed through normal channels are already coming under added pressure trom the Services. How will this be when 25 or more complete mass-radiographic units are working to full capacity all over the country? The answer is, more beds are certain to be required, and more nurses and more doctors. Even if these cannot be found at once, as clearly they cannot, it must be remembered that the pressure will come rather slowly at first as new units gradually enter the field, and there will be time for adjustment.

But even if there were no prospect of more beds, this would be no argument against massradiography. The wise general learns all he can about the enemy's dispositions. With phthisis in our midst it is safer for us to be aware of its lurking-places than to let it continue to spread silently. The very fact of diagnosis and the institution of elementary hygienic precautions are not without value. Treatment facilities must eventually arise, as the history of all social services goes to show. When the Cuban Government decided to examine by mass-radiography 
400,000 inhabitants of Havana, there was not a single sanatorium in the country, but the cases of tuberculosis discovered in this successful survey necessitated the building of sanatoria which had long been required for the known consumptives. Here in Great Britain, we are relatively well supplied with institutions for the tuberculous, and the great E.M.S. Hospital network offers obvious possibilities for post-war expansion, so there is little fear of our being suddenly swamped by cases discovered by mass-radiography.

\section{Should Tuberculosis be discovered?}

Opponents of mass-radiography, however, cast doubt not only on our ability to treat the cases we discover, but, even more insidiously, on whether it is desirable to discover them at all. Many of those with asymptomatic lesions, they argue, recover spontaneously, as the finding of healed history-free tuberculosis shows. May it not be that the early active lesion could be trusted, even if undetected, to go on to healing or resolution? If so, we not only fail to help the individual by needlessly drawing his attention to previously unsuspected disease, but may positively upset its benignity by our unjustified interference. The anxiety state imposed by an unwelcome disillusionment, aggravated by the stigma of his becoming a notified case of tuberculosis, may in fact precipitate breakdown and spread in an otherwise benign focus!

Such criticisms are characteristically imaginative and obviously come from lack of experience in this specialised work. I have personally found, in several hundred interviews arising out of various mass-radiographic surveys, a rational and objective attitude in patients which is apparently lacking in certain sections of the medical profession. Alarm and despondency occur as a rule only when the preliminary propaganda has not been properly presented, and in any case are quickly dissipated when the individual situation is explained and discussed. The stigma of tuberculosis fast loses its meaning when people learn that a considerable proportion of their number harbour tuberculous foci, and this may yet turn out to be one of the greater benefits of mass-radiography. As for notification, this need not be effected in observation cases, and can quite simply be deferred until definitely active disease is established.

While there may be no statistical proof that detection and treatment of a small early lesion ultimately improves the individual prognosis, there is not a scrap of evidence to the contrary, and surely we have long passed the nihilistic view that tuberculosis in general is not amenable to treatment. Nor can it be doubted that the small early lesion, if it sometimes heals spontaneously, may on the other hand spread into massive disease without symptomatic warning, a phenomenon amply illustrated in the follow-up of infected cases.

Early lesions, as has already been stressed, form only one group of cases detected by massradiography. Perhaps more important still are those with advanced, chronic, highly infectious phthisis known in the tuberculosis service as "source" cases, because of their capacity for maintaining a supply of secondary sufferers. In epidemiological doctrine, the detection and neutralisation of such carriers of tubercle bacilli becomes increasingly necessary as the general incidence of tuberculosis grows less. In areas of the U.S.A., where the disease has reached a level far below ours, but which we in our turn may expect it to reach if the pre-war curve of incidence were to continue in its downward trend, such cases are believed to constitute a corresponding greater menace, as they may at any time produce epidemic tuberculosis in a stock with reduced immunity.

Why then, demand the nihilists in a last sacrificial effort, do we introduce a measure aimed at abolishing the immunity acquired by non-fatal casual infection? The answer is, because such immunity is at best very incomplete and its effectiveness always uncertain; living tubercle bacilli in unknown dosage are more likely to be harmful than beneficial, and 30,000 annual deaths are too high a price to pay for a scientific half-truth. w. We still lack either a universal prophylactic or a certain permanent cure for tuberculosis: both of these may in theory be very near our grasp, in B.C.G. vaccination on the one hand and chemotherapy on the other, but neither yet fulfils our requirements. There remains only one rational means for fighting the disease on a mass scale; that is according to the classical dictum of Frost (I938) that if the ratio of new cases to deaths be kept at less than unity, tuberculosis must, like leprosy, dwindle to no more than a minor local problem.

The policy of detection and neutralisation of infectious cases of tuberculosis has operated with no little success in the past. Mass-radiography comes as a new weapon at a critical moment 
of the battle, perhaps at a moment when we need it most. We can afford to neglect no weapon, least of all one so tried and true. Let us see that it is used to the full.

\title{
REFERENCES
}

BRAILSFORD, J. F. (1943), Public Health, lvi, II7.

CHADWICK, H. D. (I927), American Rev. Twb., xv, 6oI.

FELLOWS, H. H., (1934), Amer. J. Med. Sci., clxoxvili, 553.

FROST, W. D., (1937), Amer. Journ. Public Health

SIEGAL, W. (1940), Tuberculosis in Industry, New York, p. 301.

TRAIL, R. R. (1943), Public Health, lvi, II7.

TOUSSIANT, C. H. A. (1943), N.A.P.T. Annual Conference, London.

\section{MASS RADIOGRAPHY}

\author{
By JAMES F. BRAILSFORD, M.D., PH.D., F.R.C.P., F.I.C.S. \\ (Consulting Radiologist to the City of Birmingham Hospitals; Honorary Radiologist to the \\ Queen Elizabeth Hospital, etc.)
}

In his presidential address to the Faculty of Radiology in I94I S. C. Shanks (1) stated: "The war has brought into prominence a problem which many of us have been dreaming about, and have regarded as Utopian - that of mass radiography of the chest. As there is now some chance that it may in future be adopted on a large scale, and as I have been somewhat involved in its consideration, it might be of interest to you to hear something of the present position."

This propaganda fell on suitable ground-for the Minister of Health in 1942 expressed some concern at the increase in the number of deaths from tuberculosis during the years I938-4I, i.e. from 26,176 in 1938 to 28,669 in I94I. The effect of the propaganda can be appreciated from the statements which he made in the House on June 30, I942. (2) He then indicated that we need to give greater practical effort to the necessity of early diagnosis. "We have tended tQ concentrate on treatment rather than early diagnosis, but the developments in miniature radio $\overline{0}$. graphy during the last few months seem to give us a new and useful weapon for detection-an $\$$ it was hoped that towards the end of the year we should be able to use radiographic units on a carefully planned basis. In this way I hope we shall be able to detect and treat tuberculosis in the early stages when we can, by a period of suitable treatment, restore a man to health and self-sufficiency with the shortest possible interruption of his normal life."

The medical journals, lay Press, and the wireless took up the propaganda, and for the most part refused to publish criticisms of the scheme, consequently the impression was given to the public that this miniature photography of the screen image, wrongly called miniature radiography, could be so applied. to the whole population, that all cases of tuberculosis would be recognised early, and prompt treatment would result in marked diminution if not eradication of the disease.

It was pointed out by the advocates (3) that each unit could deal with some 300 cases per hour, or " 4 per minute without undue fatigue," and that the interpretation of the miniature photographs could be done at a similar rate. Apparently no attempt was made to consider what these figures meant when applied to the population, say four times a year. The enormous number of units required and, even more important still, the number of trained medical and lay staffs and accommodation in normal times, would alone have sufficed to check the enthusiasm of any advocate. The degree to which the mentality was affected by war panic in this blackest period of our history can be measured by the fact that at the time the scheme was launched we probably had the greatest relative poverty in equipment, medical and lay personnel, and accommodation in our history. To do the whole population at three-monthly intervals, with the saner rate now advised, we should need the accommodation and personnel for something like 4,000 units. No treasury in peace time would countenance such prodigious expenditure on one aspect of public health when so many call for attention. Since the publication of previous criticism $\stackrel{Q}{\square}$ by the author (4) and (5) the "Advisory Report on the working of a Mass Radiography Unit," "to give practical advice on the establishment and operation of the scheme for mass miniature radiography" has been issued, and in this paper attention will be given to the points raised in this report of the sub-committee of the Standing Advisory Committee on Tuberculosis. 